\title{
NUEVOS INSTRUMENTOS PARA LA PROTECCIÓN DE LOS DERECHOS HUMANOS EN EL CONTEXTO DE LA GLOBALIZACIÓN: APUNTES SOBRE EL TRATADO DE EMPRESAS Y DERECHOS HUMANOS
}

\section{NEW INSTRUMENTS OF HUMAN RIGHTS PROTECTION IN THE CONTEXT OF GLOBALIZATION: NOTES ON THE BUSINESS AND HUMAN RIGHTS TREATY}

Daniel Iglesias Márquez

Universidad de Sevilla, Sevilla, España

dimarquez@us.es

Palabras clave: globalización, empresas, derechos humanos, tratado, prevención y reparación

Keywords: globalization, business, human rights, treaty, prevention and remediation

Resumen: El proceso de globalización ha creado las condiciones favorables para que las empresas expandan sus actividades y acumulen capital en un contexto sin fronteras económicas. Esto, a su vez, ha supuesto importantes retos para garantizar el disfrute de los derechos humanos y proteger el medio ambiente frente a las actividades empresariales. Esta situación ha dado lugar a diversos instrumentos cuyo objetivo es prevenir y reparar abusos y violaciones de derechos humanos cometidas por las empresas. El presente artículo reflexiona sobre el posible valor añadido de un tratado sobre empresas y derechos humanos para hacer frente al poder y a la impunidad corporativa y sobre cómo encaja un instrumento de esta naturaleza en el contexto de la globalización económica.

Abstract: Globalization has created the favourable conditions for businesses to expand their activities and to accumulate capital in a context without economic borders. This in turn has raised significant challenges for ensuring the enjoyment of human rights and environmental protection in the context

1. El presente estudio ha sido realizado en el marco del proyecto de investigación "Gobernanza y Aplicación de la Responsabilidad Social Empresarial en la Unión Europea” (DER2017-85834-R) y del Grupo de Investigación SEJ 055 "Nuevos sujetos, nuevos derechos, nuevas responsabilidades: derechos humanos en la sociedad global". 
of business activities. Against this background, various instruments have emerged whose objectives are to prevent and to ensure effective access to remedy for victims of human rights abuses and violations committed by businesses. This papers explores the possible added value of a treaty on business and human rights to deal with corporate power and impunity. It also examines how this instrument fits into the context of economic globalization.

\section{Reflexiones preliminares: globalización, empresas y derechos humanos}

Las sociedades contemporáneas han sido testigo de una dinámica de acelerados cambios cualitativos (sociales, culturales, políticos, ambientales y tecnológicos) y cuantitativos (económicos) como consecuencia del auge del proceso multifacético de la globalización. ${ }^{2}$ Durante las últimas

2. La globalización es un término difícil de concretar. Entre los tratadistas clásicos del tema no existe una definición uniforme de lo que se entiende por globalización. De la Dehesa (2000: 17), por ejemplo, la define como "un proceso dinámico de creciente libertad e integración mundial de los mercados de trabajo, bienes, servicios, tecnología y capitales". Soros (2002: 19) por su parte la equipara al "desarrollo de mercados financieros globales y al crecimiento de corporaciones transnacionales con su creciente dominio sobre las economías nacionales". Beck (1997: 29) la describe como "los procesos en virtud de los cuales los Estados nacionales soberanos se entremezclan e imbrican mediante actores transnacionales y sus respectivas probabilidades de poder, orientaciones, identidades, entramados varios". En un sentido similar, Robertson (2005: 18) define la globalización como "el conjunto de procesos que conducen a un mundo único" o décadas, las oportunidades y los riesgos de este proceso han sido objeto de intensos debates a diferentes escalas. La posición clásica de quienes la defienden es que la consideran como un proceso real y profundamente transformador capaz de producir riqueza y bienestar, ya que permite el acceso rápido y masivo a nuevas y mejores técnicas de producción de bienes y de consumo, aumentando así el bienestar económico (Vivas Agüero, 1999: 59-80). También defienden que la apertura del comercio internacional y las políticas de restructuración del capital ayudan a los países a crecer económicamente mucho más rápido, que el comercio exterior fomenta el desarrollo a través de las exportaciones y que, entre otras ventajas, reduce los índices de pobreza (Stiglitz, 2002: 3640). La mayoría de sus supuestas virtudes están asociadas a un desarrollo puramente económico. Se dice, por tanto, que se trata de un proceso de índole principalmente económico que ha internacionalizado las economías a través de la integración e interdependencia entre los Estados. Por ello, prioriza el desarrollo económico sobre el desarrollo humano mediante el aumento significativo de los avances tecnológicos, el comercio internacional, la producción y los flujos de capital (Albi Ibáñez, 2005: 9-18).

Es bien sabido que este contexto de internacionalización del capital ha favorecido la expansión de las empresas no solo en términos numéricos sino financieros en un espacio sin fronteras para la economía. La presencia y participación de estas entidades en las relaciones (comerciales y políticas) internacionales las convierte en motores y, a su vez, en beneficiarias del proceso

en otras palabras al "hecho de que las interrelaciones entre los seres humanos han adquirido proporciones globales y se han transformado en dicho proceso". 
de globalización. Sus estructuras flexibles y descentralizadas están adaptadas para ser vehículos de la internacionalización, la acumulación y la concentración del capital. De acuerdo con de la Dehesa (2000: 113), las empresas son "el principal conducto por el cual la globalización se está desarrollando y, a su vez, ésta [promueve] el rápido desarrollo de las empresas multinacionales o globales. Es decir, ambas se autoalimentan, reforzándose mutuamente".

Evidentemente el papel de las empresas ha sido clave en el proceso de globalización económica y en la expansión de las políticas neoliberales en las que han cimentado su poder económico y político (Moro, 2006: 37-53). La relevancia e influencia de estos actores en el contexto de la globalización económica ha impulsado las dinámicas de regulatory competition y race to the bottom, que empujan a los Estados, principalmente en las economías en desarrollo y emergentes, a utilizar sus estándares sociales, laborales o ambientales como factor a reducir para lograr una mayor competitividad en el mercado global y atraer la inversión extranjera. Así, las empresas operan en Estados con costes de producción más reducidos y con estándares y marcos reguladores nacionales menos exigentes (dumping), que les permiten no solo generar el máximo benéfico al menor coste y tiempo posible, sino también libre de riesgos, ya que se escudan de cualquier tipo de responsabilidad jurídica por sus impactos negativos cometidos a lo largo de las complejas estructuras empresariales y cadenas de suministro. Por tanto, las empresas operan a través de redes descentralizadas y deslocalizadas, con una clara orientación Norte-Sur, pero que se empieza a extender en horizontal (Norte-Norte o Sur-Sur), capaces de situar las distintas fases de la producción ya no solo en distintas plantas o centros de trabajo, sino en diferentes países a través de filiales o empresas subcontratadas con escasos vínculos con el territorio o el mercado local (Guamán, 2018: 100-124).

Por lo anterior, no se pueden obviar las externalidades negativas de las actividades de las empresas en el disfrute de los derechos humanos y el medio ambiente, que, particularmente en los Estados del Sur Global, afectan a los grupos en situación de vulnerabilidad y marginalidad. Son diversos los casos documentos en todo mundo en los que las empresas están implicadas en abusos de derechos humanos, derechos colectivos y derechos laborales. Por ejemplo, diversas empresas del sector extractivo han sido acusadas de afectar gravemente el medio ambiente. Esta situación vulnera el derecho a la vida, a la integridad personal y a la salud, entre otros, de las personas y de las comunidades que habitan en las inmediaciones de sus operaciones y que dependen directamente de los recursos naturales. Por su parte, las empresas del sector textil han llamado la atención de la comunidad internacional por los abusos de derechos laborales cometidos en las empresas subcontratadas en las cadenas globales de suministro, ubicadas principalmente en países del sudeste asiático, América Central y México, África del Norte y en el este de Europa. Asimismo, las empresas del sector de la tecnología y la comunicación han facilitado a diversos regímenes autoritarios equipos de vigilancia utilizados para cometer sistemáticamente actos de torturas y desapariciones forzadas en contra de personas defensoras de los derechos humanos, disidentes y opositores políticos. Finalmente, cabe hacer referencia a las empresas de la industria militar y de seguridad privada, que han estado implicadas en una serie de 
violaciones de derechos humanos e incidentes que han resultado en la pérdida de vidas humanas, principalmente en zonas afectadas por conflictos.

Los Estados, principales garantes del respeto de los derechos humanos y de la protección del medio ambiente, deben responder con acciones legales y políticas para corregir las externalidades negativas del modelo de desarrollo capitalista heteropatriarcal, impulsado por las grandes empresas ajenas a cualquier control democrático (Iglesias Márquez y Felipe Pérez, 2018: 51-53). Sin embargo, en el contexto de la globalización, los abusos de derechos humanos cometidos en el marco de las actividades empresariales son más visibles y se han agudizado en las últimas décadas. Esto se debe a que el desajuste progresivo entre los marcos institucionales del poder y los cambios en la distribución de riqueza en este mundo globalizado han limitado la capacidad regulatoria y de fiscalización de los Estados para hacer frente a la estructura flexible y descentralizada de las empresas que operan a nivel global. La menor presencia del Estado como ente regulador económico y social ha servido para reafirmar el lugar central que ocupan las empresas en el proceso de globalización.

Por otra parte, se han generado también diversos obstáculos (legales, financieros, prácticos y procedimentales) que dificultan la posibilidad de hacer efectiva la responsabilidad jurídica de las empresas por sus impactos en el disfrute de los derechos humanos y que generan una situación de indefensión para las víctimas. Dichos obstáculos se derivan de la propia estructura de las empresas, de su capacidad económica y política de defensa, de la falta de voluntad de los Estados y de la inexistencia de marcos jurídicos apropiados a nivel interno. Asimismo, en última instancia, no se debe obviar tampoco que la impunidad de las empresas se debe en gran medida a la inadecuada estructura y normatividad bajo el derecho internacional -de los derechos humanos-, que no establece obligaciones directas para las empresas y, por otra parte, los Estados -de origen- que no se hacen responsables internacionalmente de su comportamiento.

La extensión de los poderes de las empresas en el contexto de la globalización, sin las correspondientes obligaciones y responsabilidades, ha generado la necesidad, tanto a nivel nacional como internacional, de regular su actuación para asegurar el respeto de los derechos humanos y la protección del medio ambiente. A día de hoy, la promoción de una conducta empresarial responsable a nivel internacional recae principalmente en directrices y principios no vinculantes. Si bien estas iniciativas e instrumentos no vinculantes son de gran relevancia, resultan insuficientes e ineficaces por sí solos, pues su alcance y su capacidad de influir en el comportamiento de las empresas han demostrado ser limitados debido a la falta de mecanismos para asegurar su cumplimiento y de sanciones en caso de inobservancia. Esta cuestión ha dado lugar a diversas iniciativas en el ámbito nacional e internacional que apuntan a la posibilidad, jurídica y política, de adoptar nuevos instrumentos vinculantes para proteger los derechos humanos frente a las actividades empresariales y para poner fin a la impunidad corporativa.

A nivel internacional, cabe destacar la apuesta del Consejo de Derechos Humanos (CDH) de las Naciones Unidas por la elaboración de un tratado sobre empresas y derechos humanos, cuyo objetivo es regular las actividades de las empresas transnacionales y otras empresas en el derecho internacional de los derechos humanos. La 
propuesta de un instrumento internacional jurídicamente vinculante en la materia no es novedosa, sino más bien se ha desarrollado a lo largo de varias décadas pero solo recientemente ha alcanzado la aceptación necesaria por los diversos grupos de interés para dar inicio a un proceso internacional tendiente a la codificación de estándares sobre la conducta empresarial y su impacto en los derechos humanos. En este sentido, los siguientes epígrafes abordan, en primer lugar, los principales instrumentos que promueven una cultura empresarial de respeto de los derechos humanos en el marco de la globalización económica, con el fin de entender los diversos enfoques que se han adoptado para abordar el tema. En segundo lugar, se analiza de manera breve el proceso de elaboración y el potencial del tratado sobre empresas y derechos humanos como un instrumento idóneo para prevenir y reparar abusos de derechos humanos cometidos en el desarrollo de las actividades de las empresas. ${ }^{3}$

\section{La instrumentalización de la conducta empresarial responsable en el contexto de la globalización: de lo voluntario a lo obligatorio}

La responsabilidad del sector empresarial de respetar los derechos humanos y proteger el medio ambiente es uno de los princi-

3. El presente artículo se finalizó antes de la publicación de la versión revisada del Proyecto del tratado sobre empresas y derechos humanos en julio de 2019. Por ello, el análisis se centra en las disposiciones previstas en el Proyecto Cero del tratado, publicado en julio de 2018. Para un estudio comparativo entre el Proyecto Cero y la versión revisada del Proyecto, véase Iglesias Márquez (2019). pales desafíos de la globalización económica. Por ello, esta cuestión se ha debatido durante varias décadas y en diferentes instancias internacionales, regionales y nacionales. Esto ha dado lugar, a su vez, a un ecosistema o constelación de estándares nacionales e internacionales sobre la cuestión de las empresas y los derechos humanos (Kirkebø y Langford, 2018: 157-185). Debido al número considerable de iniciativas e instrumentos existentes a día de hoy para hacer frente a los impactos negativos de las empresas y al límite de espacio de la presente contribución, en este apartado se da una pincelada de los intentos de regular la conducta empresarial en virtud del derecho internacional de los derechos humanos y de los principales desarrollos alcanzados a nivel global, regional y nacional para prevenir los abusos de derechos humanos cometidos por las empresas y, en su defecto, para asegurar el derecho de las víctimas al acceso a la justicia.

Tras la tercera Conferencia de las Naciones Unidas sobre Comercio y Desarrollo, celebrada en 1972, la estrategia inicial de las Naciones Unidas se orientó a tratar de imponer a las empresas un marco normativo para prevenir y reparar las violaciones de derechos humanos que les pudieran ser atribuibles (Pigrau Solé, 2018: 27-68). Así, el primer intento para regular internacionalmente a las empresas tuvo lugar en los debates de la Asamblea General de las Naciones Unidas sobre la soberanía permanente de los recursos naturales en la década de los sesenta (GA Res. $1803(\mathrm{XVII})$ ). De esta forma, se creó la Comisión de Empresas Transnacionales de las Naciones Unidas y en 1974 se iniciaron las labores para la elaboración de un Código de Conducta para las Empresas Transnacionales (E/1988/39/Add.1). Este instrumento se elaboró en un período de 
reajustes a la economía internacional y de afirmación política del Sur Global (entre 1970 y 1990), por lo que estaba destinado en esencia a promover y crear las condiciones adecuadas de cooperación entre las empresas y los Estados. No obstante, este instrumento no prosperó por la imposibilidad de llegar a un consenso (Sauvant, 2015: 11-87).

Con un objetivo similar, en 2003, la Subcomisión de Promoción y Protección de los Derechos Humanos, órgano subsidiario de la antigua Comisión de Derechos Humanos, aprobó las Normas de las Naciones Unidas sobre Responsabilidades de las Empresas Transnacionales y otras Empresas Comerciales en la esfera de los Derechos Humanos (E/CN.4/Sub.2/2003/12/ Rev.2). En un contexto histórico distinto, las Normas se concibieron como la base para el potencial desarrollo de un tratado con obligaciones compartidas entre los Estados y las empresas de respetar los derechos humanos y prevenir violaciones a los mismos. Su objetivo, por tanto, era desarrollar estándares normativos para regular las actividades de las empresas transnacionales y otras empresas comerciales en el ámbito de los derechos humanos (Weissbrodt y Kruger, 2003: 901-922). Las Normas intentaron imponer obligaciones vinculantes a las empresas transnacionales y otras empresas comerciales y en ello residió la falta de apoyo político por parte de los Estados y de las empresas, que manifestaron fuertes reservas e hicieron hincapié en un instrumento de este tipo no debía apartarse de la estructura tradicional del derecho internacional público (De Schutter, 2016: 41-67).

Más allá de los intentos fallidos de adoptar un instrumento internacional vinculante para proteger y respetar los derechos humanos en el marco de las actividades empresariales, destacan las iniciativas volun- tarias que contienen estándares sociales y ambientales aplicables a las operaciones de las empresas. En 1976, se adoptaron las Líneas Directrices de la Organización para la Cooperación y el Desarrollo Económicos (OCDE) para Empresas Multinacionales, que contienen recomendaciones de carácter no vinculante dirigidas por los gobiernos a las empresas multinacionales. Este documento fue revisado por última vez en 2011. Asimismo, la OCDE ha elaborado recientemente una serie de guías que promueven una conducta empresarial responsable en determinados sectores y cadenas de suministro: minerales, agricultura, textil y de calzado, industrias extractivas y finanzas. Por su parte, en el seno de la Organización Internacional del Trabajo (OIT), se adoptó en 1977 la Declaración Tripartita de Principios sobre las Empresas Multinacionales y la Política Social, actualizada por última vez en 2017.

Con un planteamiento similar, pero de iniciativa privada, encontramos la Global Reporting Initiative (GRI) y las Sustainability Reporting Guidelines. Junto a estos textos de alcance general, han proliferado otros códigos sectoriales concebidos como marcos de autorregulación o como ámbitos de cooperación multistakeholder, centrados en un sector empresarial o en un ámbito de problemas más concreto, con objetivos y lógicas de funcionamiento muy variadas. Es el caso, por ejemplo, del Sistema de Certificación del Proceso de Kimberley, relativo a los diamantes procedentes de zonas en conflicto (2000); los Principios Voluntarios sobre Seguridad y Derechos Humanos en el ámbito de la seguridad de las industrias extractivas (2000); los Principios para la Inversión Responsable (2006); y el Documento de Montreux sobre las obligaciones jurídicas internacionales pertinentes y las buenas 
prácticas de los Estados con respecto a las operaciones de las empresas militares y de seguridad privadas durante los conflictos armados (2008).

Actualmente los Principios Rectores de las Naciones Unidas sobre las Empresas y los Derechos Humanos (en adelante Principios Rectores) (A/HRC/17/31) son el instrumento más relevante en la materia. Estos Principios fueron adoptados en el marco del CDH en 2011. A pesar de las críticas y el lento proceso de implementación a nivel nacional, este instrumento marcó un punto de inflexión en la agenda de las Naciones Unidas sobre el tema de empresas y derechos humanos. Asimismo, cabe destacar que se crearon a partir de un consenso histórico entre la comunidad empresarial, los Estados y la sociedad civil a nivel internacional.

Este instrumento de soft law está basado en tres pilares, que agrupan 31 principios y sus comentarios respectivos: 1) la obligación de proteger los derechos humanos por parte de los Estados, 2) la responsabilidad de las empresas de respetar los derechos humanos y 3) el acceso a la reparación por parte de las víctimas de vulneraciones de derechos humanos cometidas por empresas. Los pilares están relacionados y cada uno de ellos compila una serie de recomendaciones y propuestas de prevención y reparación que se interrelacionan y que deberían ser implementadas a nivel interno mediante la adopción de políticas, leyes y reglamentos, que tengan como finalidad que las empresas respeten efectivamente los derechos humanos y prevengan las consecuencias adversas de sus actividades y las de sus filiales y socios comerciales.

Como bien indica el profesor Ruggie, la adopción de los Principios Rectores se tra- ta de la fase final del comienzo de un proyecto que necesita continuar construyendo un andamiaje jurídico a nivel internacional para garantizar la protección y realización de los derechos humanos en el contexto de las actividades empresariales ( $\mathrm{A}$ ) HRC/17/31, párr. 13). Por tanto, los Principios Rectores han servido de plataforma para que a nivel regional y nacional se elaboren y adopten instrumentos vinculantes que regulan la conducta de las empresas y que protegen los derechos humanos frente a sus actividades. Estos desarrollos normativos han tenido lugar principalmente en el ámbito europeo y en menor medida en otras latitudes del mundo.

En el plano supranacional, cabe destacar la Directiva 2014/95/UE del Parlamento Europeo y del Consejo por la que se modifica la Directiva 2013/34/UE en lo que respecta a la divulgación de información no financiera e información sobre diversidad por parte de determinadas grandes empresas y determinados grupos. Este instrumento impone a las empresas que entran dentro del ámbito de aplicación de la norma (las que tienen más de 500 trabajadores), la obligación de preparar un informe que contenga información relativa a cuestiones de derechos, ambientales y sociales, así como sobre los procedimientos de diligencia debida aplicados por la empresa. En este mismo sentido, el Reglamento (UE) 2017/821 del Parlamento Europeo y del Consejo, por el que se establecen obligaciones en materia de diligencia debida en la cadena de suministro en lo que respecta a los importadores de la Unión de estaño, tantalio y wolframio, sus minerales y oro originarios de zonas de conflicto o de alto riesgo. Este Reglamento obliga a los importadores de la Unión Europea de minerales o metales a adoptar procesos de diligencia debida, 
según lo establecido en las Guía sectorial de la OCDE para el sector minero.

A nivel nacional cabe destacar algunos instrumentos adoptados en el Reino Unido, Francia, Australia y California, que controlan y regulan las actividades de las empresas más allá de las fronteras territoriales sin transgredir la soberanía de terceros Estados. En el Reino Unido y Australia destacan las leyes sobre esclavitud moderna que imponen a determinadas empresas la obligación de publicar cada año financiero una declaración donde se manifieste que se han tomado los pasos necesarios para evitar la esclavitud y el tráfico de personas en sus cadenas de suministro. Esta misma fórmula regulatoria se presenta de manera similar en la California Transparency in Supply Chain Act de 2010. En Francia, por su parte, se aprobó la Loi relative au devoir de viligance des sociétés mères et des entreprises donneuses d'ordre en 2017. Esta ley impone a las empresas que se encuentran bajo su ámbito de aplicación la obligación de elaborar y publicar un plan de vigilancia, que incluya las medidas razonables para identificar y prevenir los riesgos e impactos negativos sobre los derechos humanos y daños ambientales que resulten de sus actividades o de las de sus subcontratistas o proveedores con una relación comercial estable (Cossart et al., 2017: 317-323). Instrumentos similares a la ley francesa se tienen previstos en Alemania, Suiza, Países Bajos y Finlandia, que han manifestado su preocupación por el impacto de las actividades de sus empresas en el disfrute de los derechos humanos (Guamán, 2018: 112-113).

El panorama se muestra favorable hacia la adopción a nivel nacional de instrumentos para prevenir y reparar abusos de derechos humanos cometidos por las empresas. Sin embargo, la sociedad civil, la academia y diversos Estados del Sur Global siguen planteando la necesidad de adoptar un instrumento internacional vinculante en materia de empresas y derechos humanos, con el fin de cubrir las lagunas de gobernanza empresarial que se mantienen en el contexto de la globalización, así como para evitar divergencias y la fragmentación de los estándares requeridos a las empresas para respetar y proteger los derechos humanos en sus actividades globales y, sobre todo, para nivelar la asimetría normativa en favor de las empresas, que gozan de derechos, pero carecen de obligaciones en el marco del derecho internacional (Hernández Zubizarreta, 2019: 152-157).

\section{Hacia un tratado sobre empresas y derechos humanos: retos y oportunidades para prevenir y reparar abusos de derechos humanos cometidos en un contexto empresarial}

En julio 2014, el CDH adoptó la Resolución 26/9 relativa a la "Elaboración de un instrumento internacional jurídicamente vinculante sobre las empresas transnacionales y otras empresas comerciales en materia de derechos humanos" (A/HRC/ RES/26/9), a cargo de un Grupo de Trabajo Intergubernamental (GTI) de composición abierta. ${ }^{4}$ La Resolución 26/9 condujo

4. Un día después de la adopción de la Resolución 26/9, el CDH adoptó por unanimidad una segunda resolución con la que extendió el mandato del Grupo de Trabajo sobre derechos humanos y empresas transnacionales y otras em- 
al histórico proceso de elaboración de un instrumento de hard law en el ámbito de empresas y derechos humanos. Asimismo, polarizó las opiniones entre los actores de interés y abrió una vez más el debate recurrente en la materia sobre los instrumentos vinculantes y no vinculantes para regular las actividades de las empresas en virtud del derecho internacional (Ruiz Galán, 2017: 819-857; De Schutter, 2016: 41-43).

La propuesta de un tratado sobre empresas y derechos humanos responde principalmente a la necesidad y a las propuestas de los países del Sur Global y de la sociedad civil de evolucionar desde el ámbito de las normas basadas en la voluntariedad a marcos jurídicos que, entre otras cuestiones, obliguen directamente a las empresas a respetar los derechos humanos en sus actividades, donde quiera que estas se realicen, extendiendo la responsabilidad a lo largo de las cadenas de suministro (Guamán, 2018: 114). En este sentido, la Resolución 26/9 fue impulsada por Ecuador y Sudáfrica y contó con 20 votos a favor, 13 abstenciones y 14 en contra. Todos los países de la Unión Europea presentes, así como Japón y Estados Unidos, votaron en contra. La votación no solo constata la dicotomía Norte-Sur que acentúa las desigualdades en el contexto de la globalización económica, sino también es un reflejo del contexto geopolítico y económico en el que operan las empresas (Gonzalez, 2015: 151-195).

El proceso de elaboración del tratado ha tenido lugar en un ambiente de confrontación política e ideológica entre los Estados

presas por tres años. Esta resolución reafirma el compromiso del $\mathrm{CDH}$ de seguir implementando los Principios Rectores sobre las empresas y los derechos humanos (A/HRC/RES/26/22). del Sur Global, apoyados por las organizaciones de la sociedad civil, y los Estados del Norte Global, respaldados por las empresas (Cassel, 2018: 211-245; Cantú Rivera, 2017: 1200-1222). Es decir, mientras que la mayoría de los Estados del Sur Global se muestran más abiertos a la idea de un tratado que ponga a prueba los límites del derecho internacional, los Estados del Norte Global son más reticentes a la adopción de un instrumento que pueda afectar los consensos globales alcanzados en el ámbito de empresas y derechos humanos (Ruggie, 2016).

La publicación del Proyecto Cero del tratado sobre empresas y derechos humanos en julio de 2018 fue un paso importante en el complejo proceso de elaboración de dicho instrumento. Se presentó como una oportunidad histórica para que los Estados lleven a cabo por primera vez negociaciones sustantivas y constructivas sobre un instrumento internacional vinculante en materia de empresas y derechos humanos. Junto con el Proyecto Cero, el GTI publicó un Proyecto de Protocolo Facultativo en septiembre de 2018 que se basa en otros instrumentos de derechos humanos como el Protocolo Facultativo de la Convención contra la Tortura y Otros Tratos o Penas Crueles, Inhumanos o Degradantes.

El instrumento propuesto en materia de empresas y derechos humanos contiene un enfoque orientado principalmente a mejorar el acceso a mecanismos de reparación a las víctimas de abusos corporativos. Sus disposiciones están principalmente dirigidas a cubrir los vacíos jurídicos existentes en relación con los mecanismos de reparación -a nivel internacional y nacional- en caso de abusos de derechos humanos cometidos por las empresas, así como para hacer efectiva la responsabilidad jurídica de las mismas. 
Por tanto, uno de los valores añadidos del futuro instrumento, junto con su protocolo facultativo, a la agenda de la globalización económica, es que contiene disposiciones que garantizan el derecho al acceso a la justicia a las personas afectadas por las actividades de las empresas.

Por otra parte, cabe señalar que el instrumento propuesto tiene un enfoque conservador que se divide en tres amplias secciones. La primera se centra en las partes preliminares del texto, la segunda en las cuestiones de fondo y de procedimiento y, la tercera, en los posibles mecanismos de seguimiento y las disposiciones generales relacionadas con el instrumento. En términos generales, el Proyecto Cero se ciñe a aquellos puntos en los que la diplomacia en el seno de las Naciones Unidas ha alcanzado un mayor consenso entre los diversos actores de interés que participaron en las sesiones del GTI (Cantú Rivera, 2018: 25-40; Guamán, 2018: 113-122; Fasciglione, 2018: 629-661). Por ende, se aleja de posturas que alteran el statu quo y ponen a prueba las fronteras del derecho internacional de los derechos humanos.

Uno de los objetivos primordiales con los que nace la propuesta de un instrumento internacional vinculante en materia de empresas y derechos humanos es la imposición de obligaciones directas a las empresas en virtud del derecho internacional de los derechos humanos. Esto con el fin nivelar la asimetría de poder que sitúa a las empresas en una posición de ventaja y dominación sobre las personas y los Estados. El marco de la globalización económica garantiza a las empresas derechos sustantivos y de reparación a través de tratados internacionales y bilaterales de inversión y comercio, que contribuyen a crear el escenario adecuado para sus negocios y actividades transnacionales. Mientras tanto, la configuración de obligaciones de proteger y respetar los derechos humanos para las empresas en el derecho internacional carece de apoyo y voluntad política por parte de los Estados (Iglesias Márquez, 2018: 165-214). Esto se ve reflejado en el instrumento propuesto, que no contempla obligaciones directas para las empresas, ${ }^{5}$ sino más bien reafirma las obligaciones de los Estados de, por un lado, proteger y respetar los derechos humanos frente a las actividades empresariales y, por otro lado, garantizar a las víctimas recursos efectivos en caso de violaciones.

Por otra parte, algunos Estados y organizaciones de la sociedad civil conciben este futuro instrumento como una oportunidad para reafirmar la primacía de los tratados internacionales de derechos humanos sobre los distintos instrumentos del derecho internacional económico y de las inversiones. No obstante, el Proyecto Cero no incluye ninguna referencia expresa a la primacía de los instrumentos de derechos humanos que pudiera corregir el desequilibrio de facto en el régimen internacional respecto a los acuerdos de comercio e inversión. Más bien, indica de

5. La única referencia a las obligaciones de las empresas de respetar los derechos humanos es en el Preámbulo que subraya que "todas las empresas, independientemente de su tamaño, sector, contexto operacional, propiedad y estructura, deben respetar todos los derechos humanos, en particular evitando que sus propias actividades provoquen o contribuyan a provocar consecuencias negativas sobre los derechos humanos y haciendo frente a esas consecuencias cuando se produzcan". No obstante, como estipula el artículo 31 del Convenio de Viena sobre el Derecho de los Tratados, los preámbulos no crean efectos jurídicos vinculantes para las partes, sino más bien que contribuyen a la interpretación de los tratados. 
manera general que los futuros acuerdos que se negocien no contendrán disposiciones que estén en conflicto con la aplicación del futuro tratado y garantizarán la defensa de los derechos humanos.

Como se observa más adelante, una de las cuestiones con más potencial del futuro instrumento es quizás que avanza en el ejercicio de la jurisdicción adjudicativa de carácter extraterritorial por parte de los Estados. Esto tiene especial relevancia teniendo en cuenta que las empresas en la era de la globalización no conocen fronteras ni tampoco las externalidades negativas de sus actividades. Si bien el lenguaje utilizado en el Proyecto Cero del tratado no hace referencia a obligaciones extraterritoriales, varias de sus disposiciones tienen implicaciones más allá de las fronteras de un Estado. Por tanto, contribuye a reafirmar que en el marco de la globalización económica las obligaciones de los Estados de proteger los derechos humanos no terminan en sus fronteras territoriales.

En este sentido, diversos órganos creados en virtud de los tratados de derechos humanos han recomendado a algunos Estados del Norte Global la adopción de medidas para impedir que las empresas registradas en su jurisdicción cometan abusos en el extranjero. El Comité de Derechos Económicos, Sociales y Culturales, ${ }^{6}$

6. La Observación general No. 24 del Comité de Derechos Económicos, Sociales y Culturales indica de manera general que, en virtud del Pacto Internacional de Derechos Económicos, Sociales y Culturales, las obligaciones extraterritoriales surgen cuando un Estado Parte puede influir en situaciones que se producen fuera de su territorio, de conformidad con los límites impuestos por el derecho internacional, controlando las actividades de las empresas domiciliadas en su territorio y/o bajo su jurisdicción y, por lo tanto, por ejemplo, recomendó al Reino Unido la adopción de medidas legislativas y administrativas apropiadas para asegurar la responsabilidad legal de las empresas domiciliadas en su jurisdicción respecto a las violaciones de los derechos económicos, sociales y culturales en sus proyectos en el extranjero, cometidas directamente por esas empresas o resultantes de las actividades de sus filiales (E/C.12/GBR/ CO/6). Por su parte, el Comité de Derechos Humanos ha alentado a Canadá y a Alemania a que establezcan claramente la expectativa de que todas las empresas domiciliadas en su territorio o bajo su jurisdicción respeten en todas sus actividades, incluidas las que se realizan en el extranjero, la normativa de los derechos humanos de conformidad con el Pacto Internacional de Derechos Civiles y Políticos (CCPR/C/CAN/CO/6; CCPR/C/DEU/ $\mathrm{CO} / 6)$.

A pesar de que el instrumento propuesto se enmarca dentro del statu quo del derecho internacional contemporáneo, este ha generado diversas opiniones encontradas entre la sociedad civil, organizaciones internacionales, academia y organizaciones empresariales, ya que muchos de los elementos propuestos son imprecisos, incoherentes e inconsistentes en relación con otras disposiciones del mismo texto e incluso algunos son problemáticos desde la perspectiva de derechos humanos. Algunos de ellos presentan deficiencias técnicas que deben ser corregidas. Por otra parte, no queda del todo claro los derechos humanos cubiertos por el ámbito de aplicación del futuro instrumento. Esta cuestión adquiere relevancia si se tiene en cuenta que las empresas afectan un

contribuir al disfrute efectivo de los derechos económicos, sociales y culturales fuera de su territorio nacional (E/C.12/GC/24). 
gran un número de derechos humanos reconocidos en diversos instrumentos internacionales que muchas veces no están ratificados por todos los Estados. Por tanto, existe una importante divergencia entre los Estados en relación con los derechos humanos reconocidos a nivel interno e incluso a nivel internacional, ya que algunos Estados reconocen derechos que no se han cristalizado en el derecho internacional. En este sentido, el ámbito de aplicación material del futuro instrumento debe evitar formulaciones demasiado amplias que se presten a interpretaciones discrepantes. Esto con el fin de evitar problemas en su aplicación.

El instrumento propuesto a día hoy requiere de mayor discusión y clarificación para lograr que se prevengan y reparen de manera efectiva los abusos de derechos humanos cometidos por las empresas en el desarrollo de sus actividades. Asimismo, es importante abordar la incoherencia y la vaguedad que presentan las disposiciones del Proyecto Cero y las subsecuentes versiones del Proyecto, para hacer frente a las críticas de los Estados del Norte Global y otros actores que se oponen y que han obstaculizado las negociaciones que conducen a la adopción de un tratado.

La presente contribución se enfoca en dos elementos del futuro instrumento que se consideran clave y urgentes para generar un cambio en el paradigma de "business as usual" en el contexto de la globalización económica. Estos son: 1) la prevención de abusos de derechos humanos cometidos en el marco de actividades empresariales y 2 ) el derecho de las víctimas de acceso a la justicia y a la reparación efectiva, que se analizan a continuación.

\section{3.l. La prevención de abusos de derechos humanos cometidos en el marco de actividades empresariales}

Los diversos actores que participan en los períodos de sesiones del GTI coinciden en que la prevención es un elemento esencial del tratado sobre empresas y derechos humanos, con el fin de evadir los costos de complejos litigios y, sobre todo, para evitar el sufrimiento de las víctimas $(\mathrm{CDH}$, 2019; Cantú Rivera, 2018: 34; Guamán, 2018: 113-122). En este sentido, según lo contemplado en el artículo 9 del Proyecto Cero del tratado, se prevé que los Estados Parte en virtud del futuro instrumento velen por que en su legislación interna todas las personas con actividades empresariales de carácter transnacional en su territorio o bajo su jurisdicción o control respeten las obligaciones de diligencia debida.

Esta disposición recoge y reinterpreta algunos de los elementos de la diligencia debida en materia de derechos humanos contemplada en los Principios Rectores ${ }^{7}$ y desarrollada en las guías sectoriales de diligencia debida de la OCDE. La diligencia debida es un término conocido por las empresas para calcular los riesgos asociados a sus transacciones de valores y financieras (Martin-Ortega, 2013: 167-

7. El Principio 17 de los Principios Rectores señala que con el fin de identificar, prevenir, mitigar y responder de las consecuencias negativas de sus actividades sobre los derechos humanos, las empresas deben proceder con la debida diligencia en materia de derechos humanos. Este proceso debe incluir una evaluación del impacto real y potencial de las actividades sobre los derechos humanos, la integración de las conclusiones, y la actuación al respecto; el seguimiento de las respuestas y la comunicación de la forma en que se hace frente a las consecuencias negativas. 
192; Lambooy, 2010: 404-448). El Principio 17 de los Principios Rectores, por su parte, busca extender el alcance de esta herramienta metodológica de conducta, con el fin de que las empresas no solo se aseguren de cumplir con las normas nacionales, sino también para identificar, prevenir, mitigar y rendir cuentas de las consecuencias negativas de sus actividades sobre los derechos humanos. ${ }^{8}$

Los pasos clave del proceso de diligencia debida que las empresas deben adoptar en el desarrollo de sus actividades son: i) evaluar las consecuencias negativas reales o potenciales sobre los derechos humanos; ii) integrar las conclusiones de sus evaluaciones de impacto en sus funciones y procesos internos; iii) dar seguimiento a la eficacia de las medidas adoptadas; y, iv) comunicar al exterior sobre cómo se abordan los impactos. E Proyecto Cero se basa en gran medida en estos pasos, sin embargo, omite de manera expresa la integración de los resultados de las evaluaciones en las operaciones de las empresas y la comunicación con terceros interesados sobre cómo se abordan los impactos (Fasciglione, 2018: 645650). En este sentido, Guamán (2018a: 22) identifica la falta de una obligación de efectuar una política de publicidad vinculada directamente con los resultados de los mecanismos de supervisión, identificación y evaluación.

Como aspecto positivo, según lo contemplado en el Proyecto Cero, el futuro instrumento introduciría algunos nuevos elementos a la diligencia debida no re-

8. Según Ruggie, la debida diligencia es "un intento amplio y activo de descubrir los riesgos reales o potenciales que amenazan a los derechos humanos durante todo el ciclo vital de un proyecto o actividad comercial, con miras a evitar o atenuar esos riesgos" (A/HRC/11/13). cogidos en los Principios Rectores. Por ejemplo, las empresas deben realizar consultas significativas con los grupos afectados, prestando una especial atención a grupos en situación de vulnerabilidad, como las mujeres, los niños y las niñas, las personas con discapacidad, los pueblos indígenas, las personas migrantes, refugiadas y desplazadas internas (artículo 9.2.g). A su vez, deben contar con una garantía financiera para hacer frente a las posibles reclamaciones de indemnización (artículo 9.2.h). Finalmente, deben incorporar medidas de diligencia debida en todas las relaciones contractuales que impliquen actividades empresariales de carácter transnacional (artículo 9.2.a).

Uno de los aspectos relevantes que presenta el futuro tratado respecto a la prevención de abusos corporativos de derechos humanos es que cristaliza la obligación de diligencia debida para las empresas. El Proyecto Cero apuesta por la implementación del primer y segundo pilar de los Principios Rectores de manera que refuerza la interrelación y conexión entre ambos pilares. Debido al carácter no vinculante de los Principios Rectores, no existe una práctica estandarizada respecto a la implementación de la diligencia debida, sino más bien predomina una expectativa social de que las empresas adopten las medidas necesarias para prevenir abusos de derechos humanos. Por tanto, en virtud del futuro tratado, los Estados estarían obligados a introducir en la legislación interna la diligencia debida obligatoria en materia de derechos humanos como mecanismo de prevención de abusos corporativos que afectan el disfrute de los derechos humanos. El incumplimiento de las obligaciones relativas a la diligencia debida daría lugar a las responsabilidades e indemnizaciones 
correspondientes. Por tanto, se podría dar un impulso importante para avanzar en los desarrollos normativos para que los Estados prevengan los impactos negativos de las empresas domiciliadas en sus territorios o bajo su control, como los señalados anteriormente.

La posición de los Estados y de las organizaciones de la sociedad civil respecto a las disposiciones de prevención del futuro instrumento es positiva. No obstante, la discusión en torno a este elemento está relacionada con la necesidad de asegurar una mayor precisión de los requisitos de diligencia debida, así como de una mayor alineación con los conceptos y la terminología contemplada en los Principios Rectores o en las guías sectoriales de la OCDE (CDH, 2019). Esto con el fin de no afectar consensos -materiales y formales- alcanzados por los diversos actores de interés sobre los estándares que las empresas deben cumplir para prevenir y rendir cuentas de sus impactos reales y potenciales sobre los derechos humanos.

Asimismo, varios Estados han expresado que el tratado no debe excluir las pequeñas y medianas empresas de las obligaciones de diligencia debida (CDH, 2019). En este sentido, Ios Principios Rectores proporcionan un enfoque más integrador que puede ser adoptado en el futuro instrumento. Los Principios Rectores reconocen que las pequeñas y medianas empresas pueden disponer de menor capacidad $\mathrm{y}$, por tanto, sus respectivos procesos y políticas de diligencia debida adoptarán formas diferentes que sean proporcionales, entre otros factores, a su tamaño. ${ }^{9}$

9. Véase el comentario del Principio 14 que señala que las pequeñas y medianas empresas pueden disponer de menor capacidad, así como de procedimientos y estructuras de gestión más informales que las grandes empresas, de modo que
La disposición del artículo 9 del Proyecto Cero tampoco toma en consideración la complejidad de implementar la diligencia debida en zonas afectadas por conflictos ni prevé el establecimiento de un órgano independiente de supervisión para asegurar que las empresas cumplan con sus respectivas obligaciones. La vaguedad e imprecisión de los requisitos, según se encuentran en el Proyecto Cero, implica el riesgo de que los Estados adopten legislaciones divergentes que puedan generar espacios en los que las empresas puedan deslocalizar sus actividades para evitar el cumplimiento de determinados requisitos más estrictos.

\subsection{El derecho de las víctimas de acceso a la justicia y a la reparación efectiva}

Una de las aportaciones más significativas del futuro instrumento son las disposiciones que reconocen los derechos de las víctimas ${ }^{10}$ de abusos corporativos y las de

sus respectivos procesos y políticas adoptarán formas diferentes. Asimismo, explica que los medios dispuestos por una empresa para cumplir con su responsabilidad de respetar los derechos humanos también pueden variar en función de si actúa, y en qué medida, a través de un grupo empresarial o a título individual. Sin embargo, la responsabilidad de respetar los derechos humanos se aplica plenamente y por igual a todas las empresas.

10. Según el artículo 4 del Proyecto Cero del tratado, se entenderá por "víctima" a toda persona que sostiene haber sufrido daños, individual o colectivamente, incluidas lesiones físicas o mentales, sufrimiento emocional, pérdidas económicas o menoscabo sustancial de sus derechos humanos, que comprenden los derechos ambientales, como consecuencia de acciones u omisiones en el contexto de actividades empresariales de carácter transnacional. El término "víctima" 
responsabilidad jurídica de las empresas, ya que estas están dirigidas a adaptar los sistemas judiciales nacionales a las prácticas económicas y comerciales contemporáneas. A través de estas disposiciones se busca eliminar o reducir los obstáculos prácticos y legales que las víctimas enfrentan para acceder a recursos judiciales y para hacer efectiva la responsabilidad de las empresas. En este sentido, uno de los objetivos previstos para el futuro instrumento es asegurar el acceso efectivo a la justicia y el recurso a las víctimas de violaciones de derechos humanos en el contexto de las actividades empresariales de carácter transnacional, e impedir que se produzcan estas violaciones.

El Proyecto Cero del futuro instrumento incluye una disposición (artículo 8) que se inspira en la obligación de los Estados de garantizar a todas las personas el acceso justo, efectivo y rápido a la justicia y a los recursos efectivos ante los tribunales nacionales competentes. ${ }^{11}$ Esta disposición enlista algunos de los siguientes derechos para las víctimas: i) acceso equitativo, efectivo y rápido a la justicia y a los recursos (restitución, compensación, reha-

también comprenderá a la familia inmediata o las personas a cargo de la víctima directa y a las personas que hayan sufrido daños al intervenir para prestar asistencia a víctimas en peligro o para impedir la victimización.

11. El derecho a un recurso efectivo ante los tribunales nacionales competentes está contemplado en los principales instrumentos de derecho humanos: artículo 8 de la Declaración Universal de Derechos Humanos; artículo 2 del Pacto Internacional de Derechos Civiles y Políticos; artículo 7 de la Carta Africana de Derechos Humanos y de los Pueblos; artículo 25 de la Convención Americana de Derechos Humanos; artículo 13 del Convenio Europeo para la Protección de los Derechos Humanos y de las Libertades Fundamentales. bilitación, satisfacción y garantías de no repetición); ii) acceso adecuado a la información pertinente para la tramitación de los recursos; iii) asistencia jurídica adecuada y efectiva durante todo el proceso legal; iv) acceso a medios diplomáticos y consulares apropiados; y iv) derecho a gozar de una consideración y atención especiales, para que los procedimientos destinados al acceso a la justicia y los recursos no den lugar a una nueva victimización.

Además de los derechos señalados anteriormente, llaman la atención las disposiciones que tienen como objetivo nivelar la asimetría de recursos en los casos de empresas y derechos humanos. Por lo general, las víctimas de abusos corporativos no cuentan con los recursos económicos necesarios para asumir los costes de los complejos y largos procesos judiciales. Por ello, se contempla la exención de los costes del litigio en el futuro instrumento. El artículo 8.5.d del Proyecto Cero señala que en ningún caso se requerirá a las víctimas que reembolsen los gastos judiciales de la otra parte de la reclamación. Si bien esta disposición contribuye a disminuir algunos de los posibles obstáculos financieros que enfrentan las víctimas, también puede generar el riesgo de crear una carga de trabajo excesiva a los tribunales nacionales por el posible aumento de casos frívolos y carentes de fundamento que no tienen oportunidad de éxito en sede judicial (Fasciglione, 2018: 645-650). En este sentido, el tratado debe establecer de manera clara los supuestos en los que se exime a las víctimas de los costes judiciales.

Asimismo, se contempla un Fondo Internacional para las Víctimas de manera similar al fondo fiduciario contemplado en el artículo 79 del Estatuto de Roma en beneficio de las víctimas de crímenes de la 
competencia de la Corte Penal Internacional y de sus familias. El funcionamiento de este fondo se definirá una vez entrado en vigor el tratado. No obstante, las delegaciones han manifestado cierta reticencia al Fondo, por la posibilidad de que sean los mismos Estados quienes asuman la carga de resarcir los abusos cometidos por las empresas (CDH, 2019).

Otras de las deficiencias que presenta la disposición contemplada en el artículo 8 del Proyecto Cero es la falta de garantías especiales para abordar la situación de las personas defensoras de los derechos humanos que se oponen a los megaproyectos de las empresas. Tampoco se contemplan los colectivos o grupos que se ven especialmente afectados por las violaciones de derechos humanos cometidas por empresas o que tienen mayores dificultades para el acceso a la justicia. En este sentido, se sugiere que este artículo integre una perspectiva de género y una referencia expresa a la protección de las personas defensoras de derechos humanos (Guamán, 2018a: 32-33; Deva, 2018; $\mathrm{CDH}, 2019)$.

Por su parte, el artículo 10 del Proyecto Cero constituye una de las disposiciones fundamentales -y a su vez controversiales- del futuro instrumento, ya que aborda la responsabilidad jurídica de las empresas en caso de abusos de derechos humanos. En virtud del futuro instrumento, los Estados Parte deberán garantizar, por medio de su derecho interno, que se exijan responsabilidades de índole penal, civil o administrativa a las personas físicas y jurídicas por violaciones de derechos humanos cometidas en el contexto de actividades empresariales.

En materia civil, se contempla que este futuro instrumento intente brindar una solución a la responsabilidad jurídica de la empresa matriz por los abusos cometidos por sus filiales o entidades de la cadena de suministro. El levantamiento del velo corporativo es una de las cuestiones que los Principios Rectores no han podido brindar orientaciones concretas (De Schutter, 2016: 47-54). El artículo 10.6 del Proyecto Cero del futuro instrumento contiene los supuestos en que una empresa matriz puede incurrir en responsabilidad por los perjuicios causados por las violaciones de los derechos humanos que surjan de las operaciones que controla o de aquellas en las que mantenga una relación suficientemente estrecha con la filial o entidad en su cadena de suministro. Asimismo, puede incurrir en responsabilidad cuando haya previsto o se debieran haber previsto riesgos de violaciones de los derechos humanos en su cadena de actividad económica. A pesar del avance que representa esta disposición, requiere una mayor claridad y precisión, ya que introduce términos usados por primera vez en todo el Proyecto Cero, como "control" o "relación suficientemente estrecha", sin proporcionar una definición. La falta de una delimitación de estos conceptos puede permitir la elusión de responsabilidades por parte de las empresas matrices respecto de las violaciones cometidas por sus filiales (Guamán, 2018a: 23-27). Sobre todo, puede llegar a resultar un incentivo perverso que evite que las matrices influyan en las actividades de sus filiales por el riesgo legal de incurrir en una responsabilidad en caso de daños (Fasciglione, 2018: 649).

Otra de las cuestiones más debatibles en torno al futuro instrumento es la responsabilidad penal de las empresas contemplada en el artículo 10.8 del Proyecto Cero, que estipula que los Estados Parte dispon- 
drán medidas, con arreglo a su derecho interno, para establecer la responsabilidad penal de todas las personas con actividades empresariales. Esta disposición ha sido considerada como un paso hacia adelante que debe mantenerse hasta el final, a pesar de que está formulada de manera flexible y que requiere de mayor precisión para alcanzar los objetivos del tratado y, sobre todo, para evitar confusiones (López Hurtado, 2018). En principio, la disposición de este artículo parece imponer la obligación a los Estados de adoptar medidas para establecer la responsabilidad penal de todas las personas con actividades empresariales de carácter transnacional, a pesar de que aún hay Estados que no reconocen la responsabilidad penal de las jurídicas en sus ordenamientos jurídicos internos (Iglesias Márquez, 2018a). No obstante, el artículo 10.12 introduce una vía de escape, ya que indica que en caso de que en el ordenamiento jurídico de un Estados Parte la responsabilidad penal no sea aplicable a las personas jurídicas, ese Estado velará por que estas estén sujetas a sanciones eficaces, proporcionadas y disuasorias de carácter no penal.

La disposición sobre la responsabilidad jurídica de las empresas contiene orientaciones sobre el desarrollo de la responsabilidad civil y penal de las empresas por violaciones de los derechos humanos. Sin embargo, no contiene disposiciones específicas que orienten a los Estados a exigir la responsabilidad administrativa de las empresas por abusos de derechos humanos. En este sentido, se obvia el potencial de algunas sanciones administrativas, como puede ser la exclusión de las empresas de participar en procedimientos de contratación pública, de acceder a subvenciones estatales o créditos a la exportación y de obtener garantías públicas para inversio- nes o créditos a la exportación. Tampoco se consideran los mecanismos no judiciales como los Puntos de Contacto Nacional de la OCDE o las instituciones nacionales de derechos humanos que pueden presentar algunas ventajas en el ámbito de sus respectivas competencias para garantizar otra vía de acceso a la reparación para las víctimas. En este sentido, cabe destacar que también es importante que las empresas cuenten con mecanismos internos, o bien, que participen en mecanismos externos, para facilitar una identificación temprana de sus potenciales riesgos e impactos negativos sobre los derechos humanos. Este tipo de mecanismos de reparación merecen una mayor atención y desarrollo en el tratado sobre empresas y derechos humanos.

\section{Reflexiones finales}

El proceso de globalización no solo ha dado lugar a nuevas formas de producción deslocalizadas y descentralizadas que benefician principalmente a los agentes económicos (empresas), sino también ha generado nuevos escenarios y formas de vulneración de derechos humanos y de degradación ambiental a gran escala. El escenario político, social y económico de la globalización implica, por tanto, retos importantes para la salvaguarda de los derechos individuales, colectivos y libertades fundamentales. En este sentido, el régimen internacional de los derechos humanos parece quedarse desfasado ante situaciones no contempladas al momento de formularse y configurarse tras la Segunda Guerra Mundial.

Lo anterior plantea la necesidad de una evolución progresista del derecho internacional de los derechos humanos que vaya 
acompañada de instrumentos y mecanismos novedosos que hagan frente a las realidades del contexto contemporáneo, como son las externalidades negativas de las actividades empresariales en la esfera de los derechos humanos. De hecho, la compleja relación entre las empresas y los derechos humanos ha dado lugar a un universo de instrumentos que orbitan entre lo voluntario y lo obligatorio para proteger y promover los derechos humanos en el marco de las actividades empresariales y asegurar el acceso a la justicia a las víctimas en caso de violaciones. No obstante, la balanza se ha declinado principalmente hacia los instrumentos voluntarios de autorregulación en el marco de la responsabilidad social corporativa, o bien, hacia los instrumentos normativos con meros requisitos formales para las empresas pero que no dan respuesta a los abusos de derechos humanos y, mucho menos, a la reparación efectiva de las víctimas. La proliferación de estos instrumentos se debe principalmente a que casan mejor con la lógica de la globalización tendiente a la desregulación y a promover una mayor competitividad de los diversos agentes que intervienen en el mercado global. Si bien estos instrumentos han creado una mayor sinergia entre los Estados y gobiernos para intentar configurar modelos y prácticas empresariales más respetuosas con su entorno y más competitivas, se ha demostrado sobradamente que por sí solos resultan insuficientes para evitar la vulneración de derechos humanos y acabar con la impunidad corporativa.

La necesidad urgente de un cambio de paradigma del "business as usual" pone cada vez menos en duda la viabilidad política y jurídica de un tratado sobre empresas y derechos humanos con la doble función de prevenir y reparar las violaciones cometidas en el marco de las actividades globales de las empresas. Un nuevo instrumento en la materia no debe concebirse como una solución holística para abordar todas las cuestiones planteadas en la compleja relación entre las empresas y los derechos humanos, sino más bien como un instrumento normativo adicional en el complejo universo de instrumentos que debe complementar y coexistir con otras iniciativas que tienen como objetivo común asegurar la responsabilidad de las empresas de respetar y promover los derechos humanos en el marco de sus actividades.

No obstante, no se debe obviar que la suerte de este futuro instrumento depende de la voluntad político-diplomática en el seno de las Naciones Unidas y, sobre todo, en qué medida este tratado encaja en el marco de la globalización económica. Un instrumento de la naturaleza del tratado sobre empresas y derechos humanos que afecta algunos de los beneficios de los principales agentes de la globalización genera fuerte oposiciones de las élites económicas y políticas. Se trata de un instrumento que intenta revindicar los derechos humanos frente a los intereses privados y, por ello, no son pocos los detractores que ven peligrar sus beneficios y libertades económicas.

Si bien se trataría de un instrumento enmarcado dentro de las fronteras del derecho internacional contemporáneo, sí que rompería varios de los esquemas en los que se sustenta la arquitectura internacional de la impunidad corporativa. El futuro instrumento pretende abordar algunos de los retos de la prevención y reparación de violaciones de derechos humanos derivados de las complejas estructuras y actividades transnacionales de las empresas, 
con el fin de asegurar la vigencia de los derechos humanos, sobre todo el derecho al acceso a la justicia de las personas afectadas por las actividades empresariales. En este sentido, es un instrumento con el potencial de alzar las voces y empoderar a las víctimas de abusos corporativos.

En suma, la adopción de un nuevo instrumento internacional vinculante en materia de empresas y derechos humanos podría, por un lado, cambiar las dinámicas de los principales actores económicos y, por otro lado, crear el contrapeso necesario a la instrumentalidad que se ha generado a partir del contexto de la globalización, cuyo diseño está pensado para afectar las facultades soberanas de los Estados y beneficiar y blindar a las élites económicas. Este instrumento se encuadra dentro de un movimiento social contra-hegemónico de resistencia para reivindicar el derecho internacional de los humanos, que se ha visto superado por el modelo económico global, con el fin de defender y proteger de manera efectiva los derechos humanos en un mundo globalizado.

\section{Bibliografía}

Albi Ibáñez, Emilio (2005), "La globalización económica como marco de las relaciones internacionales", en Revista ICE, núm. 825, España: Secretaria de Estado de Comercio.

Beck, Ulrich (1997), ¿Qué es la globalización? Barcelona-Buenos Aires-México: Paídos.

Cantú Rivera, Humberto (2017), "Negotiating a Treaty on Business and Human Rights: The Early Stages", en University of New South Wales Law Journal vol. 40, núm. 3: Sydney: University of New South Wales.
Cantú Rivera, Humberto (2018), "Some remarks on the third sessions of the Business and Human Rights Treaty Process and the Zero Draft", en Brazilian Journal of International Law, vol. 15, núm. 2, Brasilia: Centro Universitario de Brasilia.

Cassel, Douglass (2018), "Opciones para un tratado sobre empresas y derechos humanos. Avances y estado del arte", en von Bogdandy, Armin, Salazar Ugarte, Pedro, Morales Antoniazzi, Mariela y Ebert Franz Christian [coords.], El constitucionalismo transformador en América Latina y el derecho económico internacional. De la tensión al diálogo, Ciudad de México: UNAM-IIJ.

CDH (2019), "Informe del Grupo de Trabajo intergubernamental de composición abierta sobre las empresas transnacionales y otras empresas con respecto a los derechos humanos sobre su cuarto período de sesiones. Disponible en: http://ap.ohchr.org/documents/dpage_e. aspx?si=A/HRC/40/48 [8 de junio 2019].

Cossart, Sandra, Chaplier, Jérôme y Beau de Lomenie, Tiphaine (2017), "The French Law on Duty of Care: A Historic Step towards Making Globalization Work for All", en Business and Human Rights Journal vol. 2, núm. 2, Cambridge: CUP.

de la Dehesa, Guillermo (2000), Comprender la globalización, Madrid: Alianza Editorial.

De Schutter, Olivier (2016), "Towards a New Treaty on Business and Human Rights", en Business and Human Rights Journal vol. 1, núm. 2, Cambridge: CUP.

Deva, Surya (2018), "The Zero Draft of the Proposed Business and Human Rights Treaty, Part II: On the Right Track, but Not Ready Yet". Disponible en: https:// www. business-humanrights.org/en/thezero-draft-of-the-proposed-business-andhuman-rights-treaty-part-ii-on-the-righttrack-but-not-ready-yet [8 de junio 2019]. 
Fasciglione, Marco (2018), "Another Step on the Road? Remarks on the Zero Draft Treaty on Business and Human Rights", en Diritti Umani e Diritto Internazionale vol. 12, núm. 3, pp 629-661.

Gonzalez, Carmen (2015), "Environmental Justice, Human Rights, and the Global South", en Santa Clara Journal of International Law, vol. 13, Santa Clara: Santa Clara University's School of Law.

Guamán, Adoración (2018), "Empresas transnacionales y derechos humanos: acerca de la necesidad y la posibilidad de la adopción de un Instrumento Jurídicamente Vinculante (Binding Treaty)", en Jueces para la democracia, núm. 92, 2018.

Guamán, Adoración. (2018a), "El Draft 0 del Binding Treaty: análisis crítico del contenido del texto y su adecuación con el objetivo de la Resolución 26/9", en Cadernos de Pesquisa Homa vol. 1, núm. 6, Brasil: Universidad Federal de Juiz de Fora.

Hernández Zubizarreta, Juan (2009), Las empresas transnacionales frente a los derechos humanos: Historia de una asimetría normativa. De la responsabilidad social corporativa a las redes contra hegemónicas transnacionales, Bilbao-Vitoria/ Gasteiz: Hegoa-OMAL.

Iglesias Márquez, Daniel (2018), "Explorando las fronteras del Derecho internacional de los derechos humanos: hacia la adopción de un tratado internacional sobre empresas y derechos humanos" en Akademía. Revista Internacional y Comparada de Derechos Humanos, vol. 1, núm. 1, Coahuila: Academia Interamericana de Derechos Humanos.

Iglesias Márquez, Daniel (2018a), "La responsabilidad penal de las empresas por graves violaciones de derechos humanos: práctica actual y desafíos futuros", en Brazilian Journal of International Law vol. 15, núm. 2, Brasilia: Centro Universitario de Brasilia.

Iglesias Márquez, Daniel (2019), "Hacia la adopción de un tratado sobre empresas y derechos humanos: viejos debates, nuevas oportunidades", en Revista Deusto de Derechos Humanos, núm. 4, Bilbao: Instituto de Derechos Humanos Pedro Arrupe.

Iglesias Márquez, Daniel y Felipe Pérez, Beatriz (2018), "Organizaciones de la sociedad civil vs. Empresas transnacionales. Estrategias frente al poder corporativo", en Pueblos - Revista de Información y Debate, núm. 77, España: Paz con Dignidad.

Kirkebø, Tori y Langford, Malcolm (2018), "The Commitment Curve: Global Regulation of Business and Human Rights", en Business and Human Rights Journal, vol. 3, núm. 2, Cambridge: CUP.

Lambooy, Tineke (2010), "Corporate Due Diligence as a Tool to Respect Human Rights", en Netherlands Quarterly of Human Rights, vol. 28, núm. 3, Utrecht: The Netherlands Institute of Human Rights.

López Hurtado, Carlos (2018), "Hacia una Convención Internacional sobre Empresas y Derechos Humanos", Disponible en: https://dplfblog.com/2018/10/04/hacia-una-convencion-internacional-sobreempresas-y-derechos-humanos/ [8 de junio 2019].

Martin-Ortega, Olga (2013), "La diligencia debida de las empresas en materia de derechos humanos: un nuevo estándar para una nueva responsabilidad", en Zamora Cabot, Francisco, García Cívico, Jesús y Sales Pallarés, Lorena [eds.], La Responsabilidad de las Empresas Multinacionales por Violaciones de los Derechos Humanos, Alcalá de Henares: Universidad de Alcalá.

Moro, Braulio (2006), "Las empresas transnacionales como agentes centrales 
de la globalización neoliberal, elementos de discusión para una propuesta de políticas alternativas", en Lan Harremanak especial. Revista de relaciones laborales, núm. Especial, España: Universidad del País Vasco.

Pigrau Solé, Antoni (2018) "Empresas multinacionales y derechos humanos: la doble vía del Consejo de Derechos Humanos de las Naciones Unidas", en Marullo, Maria Chiara y Cabot, Francisco Zamora [eds.], Empresas y Derechos Humanos. Temas actuales, Nápoles: Editoriale Scientifica.

Robertson, Robbie (2005), Tres olas de globalización, Madrid: Alianza ensayo.

Ruggie, John (2016), "Incorporating human rights: lessons learnt, and next steps", en Baumann-Pauly, Dorothée y Nolan, Justine [eds.], Business and Human Rights: From Principles to Practice, London: Routledge.

Ruiz Galán, Juan (2017), "Búsqueda de Consensos Sobre Empresas y Derechos Humanos: Hacia un Instrumento Internacional Vinculante", en American University International Law Review, vol. 32, núm. 4, Cambridge: CUP.

Sauvant, Karl (2015), "The Negotiations of the United Nations Code of Conduct on Transnational Corporations. Experience and Lessons Learned", en Journal of World Investment \& Trade, vol. 16, núm. 1, Leiden: Brill.

Soros, George (2002), Globalización, Barcelona: Planeta.

Stiglitz, Joseph (2002), El malestar de la globalización, Madrid: Punto de lectura.

Vivas Agüero, Pedro (1999), "Globalización de la economía y/o mundialización del capital", en Revista de la Facultad de Ciencias Económicas, núm. 11, Perú: Universidad Nacional Mayor de San Marcos.

Weissbrodt, David y Kruger, Muria (2003), "Norms on the Responsibilities of Trans- national Corporations and Other Business Enterprises with Regard to Human Rights", en American Journal of International Law, vol. 97, núm. 4, Cambridge: CUP. 